Determination of toxic elements and arsenic species in salted foods and sea-salt by ICPMS and HPLC-ICP-MS

In Min Hwang ${ }^{\mathrm{a}}$, Hee Min Lee ${ }^{\mathrm{b}}$, Hae-Won Lee ${ }^{\mathrm{a}}$, Ji-Hye Jung ${ }^{\mathrm{b}}$, Eun Woo Moon ${ }^{\mathrm{b}}$, Naeem Khan ${ }^{\mathrm{c}}$, Sung Hyun Kim ${ }^{\mathrm{b} *}$

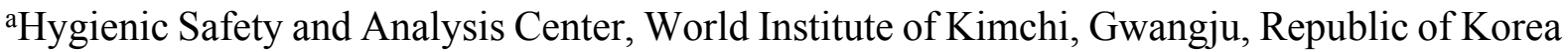
bStrategy and Planning Division/SME Service Department, World Institute of Kimchi, Gwangju, Republic of Korea

'Department of Chemistry, Kohat University of Science and Technology, Kohat 26000, Khyber Pakhtunkhwa, Pakistan

* Corresponding author:

E-mail:shkim@wikim.re.kr

Tel: $+82-62-610-1731$

Address: World Institute of Kimchi, 86, kimchi-ro, Nam-gu, Gwangju, 61755, Republic of Korea

E-mail addresses: imhwang@wikim.re.kr (I. M. Hwang), hmlee@wikim.re.kr (H. M. Lee), lhw0875@wikim.re.kr (H. Lee), luneeo@wikim.re.kr (E. W. Moon), naeemkhankust@gmail.com (N. Khan) 
Table S1. ICP-MS operating conditions for determination of toxic elements.

\section{ICP-MS condition}

Instrument

300D (Perkin Elmer SCIEX, CT, USA)

Nebulizer

Meinhard nebulizer with cyclonic spray chamber

RF power $(\mathrm{kW})$

1.35

Argon flows (L/min)

Plasma (16.00), Auxiliary (1-1.3), Nebulizer (1-1.07)

Analysis mode

DRC mode, $\mathrm{O}_{2} 0.5 \mathrm{~mL} / \mathrm{min}$

Analytical mass (Isotope)

$\mathrm{AsO}^{+}(91), \mathrm{Cd}(111), \mathrm{Pb}(208)$

Optimisation (Isotope)

On masses $\mathrm{Be}(9), \mathrm{Co}(59), \operatorname{In}(115)$, and $\mathrm{U}(238)$ 
Table S2. HPLC-ICP-MS operating conditions for determination of arsenic species.

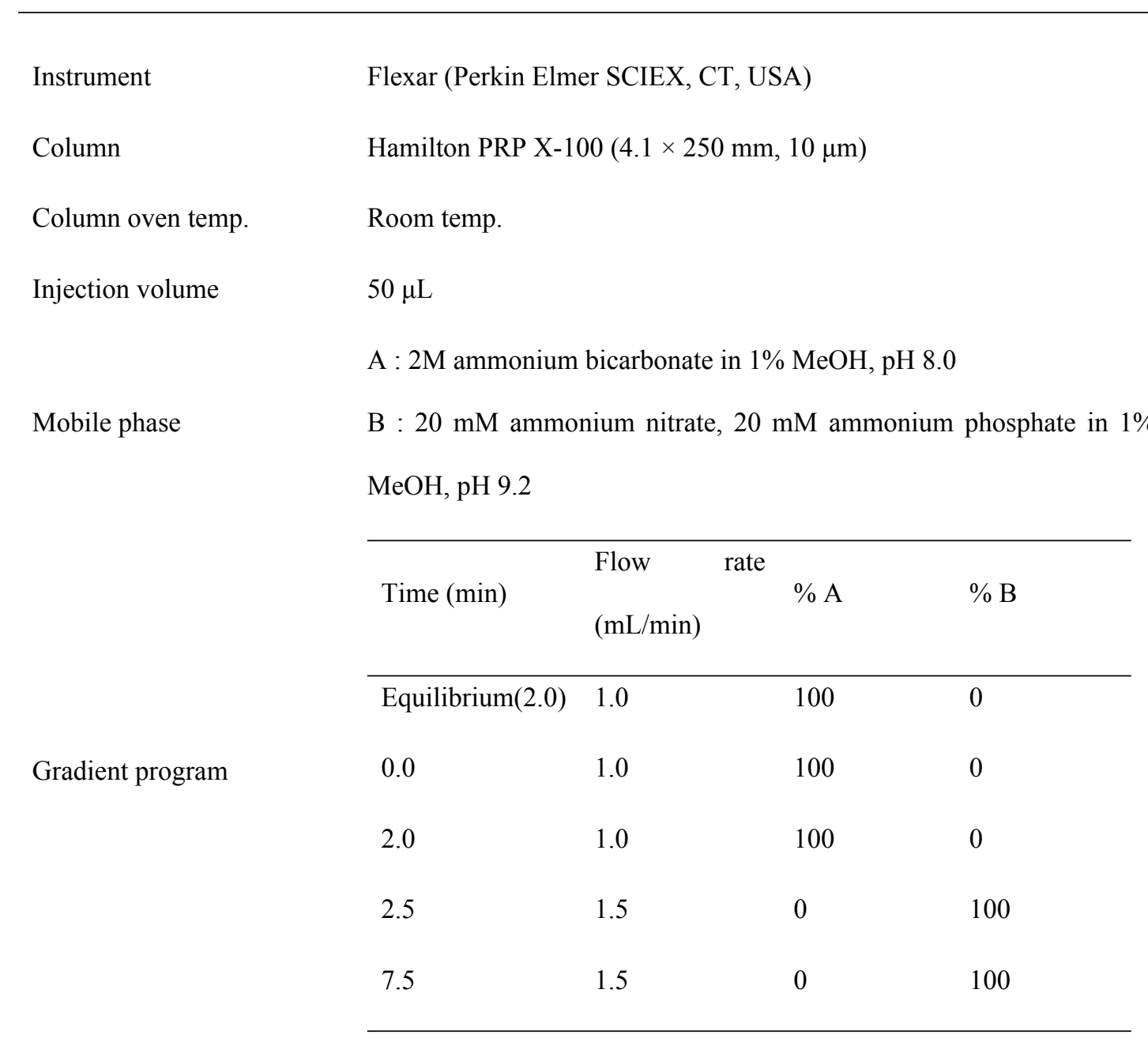


Table S3. Concentrations ( $\mathrm{mg} / \mathrm{kg}$ ) of arsenic species in seawater based on sea salt.

\begin{tabular}{|c|c|c|c|c|c|}
\hline & Korea & Vietnam & China & Australia & New Zealand \\
\hline \multicolumn{6}{|c|}{ Organic Arsenic } \\
\hline $\mathrm{AsC}$ & $<\mathrm{DL}^{\mathrm{A}}$ & $<\mathrm{DL}$ & $<\mathrm{DL}$ & $<\mathrm{DL}$ & $<\mathrm{DL}$ \\
\hline \multirow{2}{*}{$\mathrm{AsB}$} & $0.002^{\mathrm{ns} \mathrm{B}} \pm 0.0005^{\mathrm{C}}$ & $0.002^{\mathrm{ns}} \pm 0.0001$ & $0.002^{\mathrm{ns}} \pm 0.0001$ & $0.002^{\mathrm{ns}} \pm 0.0002$ & $0.002^{\mathrm{ns}} \pm 0.0001$ \\
\hline & $(0.002-0.003)$ & $(0.001-0.002)$ & $(0.002-0.002)$ & $(0.002-0.002)$ & $(0.002-0.002)$ \\
\hline DMA & $<\mathrm{DL}$ & $<\mathrm{DL}$ & $<\mathrm{DL}$ & $<\mathrm{DL}$ & $<\mathrm{DL}$ \\
\hline MMA & $<\mathrm{DL}$ & $<\mathrm{DL}$ & $<\mathrm{DL}$ & $<\mathrm{DL}$ & $<\mathrm{DL}$ \\
\hline \multicolumn{6}{|c|}{ Inorganic Arsenic } \\
\hline AsIII & $<\mathrm{DL}$ & $<\mathrm{DL}$ & $<\mathrm{DL}$ & $<\mathrm{DL}$ & $<\mathrm{DL}$ \\
\hline As V & $<\mathrm{DL}$ & $<\mathrm{DL}$ & $<\mathrm{DL}$ & $<\mathrm{DL}$ & $<\mathrm{DL}$ \\
\hline
\end{tabular}

${ }^{\mathrm{A}} \mathrm{DL}:$ below detection limit

${ }^{\mathrm{B}} \mathrm{ns}$ : non significantly differ within a row $(p<0.05)$.

${ }^{\mathrm{C}}$ Mean value \pm standard deviation 